\title{
Qualitative and Quantitative Analysis of Volatile Components of Zhengtian Pills Using Gas Chromatography Mass Spectrometry and Ultra-High Performance Liquid Chromatography
}

\author{
Cui-ting Liu, ${ }^{1}$ Min Zhang, ${ }^{1}$ Ping Yan, ${ }^{1}$ Hai-chan Liu, ${ }^{2}$ Xing-yun Liu, ${ }^{1}$ and Ruo-ting Zhan ${ }^{1}$ \\ ${ }^{1}$ Research Center of Chinese Medicinal Resource Science and Engineering, Key Laboratory of Chinese Medicinal Resources from \\ Lingnan of Ministry of Education, Joint Laboratory of National Engineering Research Center for the Pharmaceutics of \\ Traditional Chinese Medicines, Guangzhou University of Traditional Chinese Medicine, Guangzhou, Guangdong 510006, China \\ ${ }^{2}$ School of Chinese Herbal Medicine, Guangzhou University of Chinese Medicine, Guangzhou, Guangdong 510006, China
}

Correspondence should be addressed to Ping Yan; ycyyp22@hotmail.com and Ruo-ting Zhan; ruotingzhan@vip.163.com

Received 10 October 2015; Revised 23 December 2015; Accepted 24 December 2015

Academic Editor: Mohamed Abdel-Rehim

Copyright (c) 2016 Cui-ting Liu et al. This is an open access article distributed under the Creative Commons Attribution License, which permits unrestricted use, distribution, and reproduction in any medium, provided the original work is properly cited.

\begin{abstract}
Zhengtian pills (ZTPs) are traditional Chinese medicine (TCM) which have been commonly used to treat headaches. Volatile components of ZTPs extracted by ethyl acetate with an ultrasonic method were analyzed by gas chromatography mass spectrometry (GC-MS). Twenty-two components were identified, accounting for $78.884 \%$ of the total components of volatile oil. The three main volatile components including protocatechuic acid, ferulic acid, and ligustilide were simultaneously determined using ultra-high performance liquid chromatography coupled with diode array detection (UHPLC-DAD). Baseline separation was achieved on an XB-C18 column with linear gradient elution of methanol- $0.2 \%$ acetic acid aqueous solution. The UHPLC-DAD method provided good linearity $\left(R^{2} \geq 0.9992\right)$, precision (RSD $<3 \%$ ), accuracy $(100.68-102.69 \%)$, and robustness. The UHPLC-DAD/GC-MS method was successfully utilized to analyze volatile components, protocatechuic acid, ferulic acid, and ligustilide, in 13 batches of ZTPs, which is suitable for discrimination and quality assessment of ZTPs.
\end{abstract}

\section{Introduction}

In comparison with conventional fully porous particle columns, core-shell particle columns have higher column efficiency and sensitivity, achieve better separation, and remain constant over a wider linear range. In addition, the columns improve the speed of the mobile phase, reduce analysis time, and improve throughput. Moreover, the backpressure produced by the columns at optimum linear velocity is very low $(<400 \mathrm{bar})$. So we take advantage of this new column to develop an efficient ultra-high performance liquid chromatography (UHPLC) method [1-4]. Gas chromatography mass spectrometry (GC-MS) has been employed in a broad range of analytical applications due to its high sensitivity and capacity to separate compounds effectively. GC-MS is commonly used to characterize and identify volatile organic compounds in complex mixtures [5-10]. GC-MS has become a popular and useful analytical tool in research on herbal medicines, especially in establishing chromatographic fingerprints for quality control of traditional Chinese medicines [11, 12], such as Fructus Xanthii [13].

Zhengtian pills (ZTPs) are Chinese patent medicine comprised of 15 medicinal herbs: Caulis Spatholobi, Radix Angelicae Sinensis, Rhizoma Chuanxiong, Asari Radix et Rhizoma, Uncariae Ramulus Cum Uncis, Paeoniae Radix Alba, Radix Rehmanniae, Radix Angelicae Dahuricae, Radix Saposhnikoviae, Notopterygii Rhizoma et Radix, Persicae Semen, Carthami Flos, Radix Angelicae Pubescentis, Ephedrae Herba, and Aconiti Lateralis Radix Praeparata [14]. ZTPs are used to treat tension headaches [15], headaches associated with spinal 
conditions, and premenstrual headaches. ZTPs are widely used in China to treat migraine headaches, a type of vascular headache $[16,17]$. According to the Chinese Pharmacopoeia, paeoniflorin is the marker compound used for ZTP quality control [14].

In a previous study, HPLC was used to determine the major active components of ZTP, which included paeoniflorin, ferulic acid, prim-O-glucosylcimifugin, and $4^{\prime}-\mathrm{O}-$ beta-glucopyranosyl-5-O-methylvisamminol [18]; however, determination of these compounds is not sufficient for comprehensive quality control of ZTPs, which contain complex bioactive constituents, including volatile components of essential oils, alkaloids, flavonoids, and coumarin compounds. Several compounds from ZTPs ingredients, including protocatechuic acid from Spatholobi Caulis, and ferulic acid and ligustilide from Rhizoma Chuanxiong and Radix Angelicae Sinensis, have pharmacological activities, including antibacterial, anti-inflammatory, and analgesic effects, as well as protective effects on the cardiovascular system [1921]. Therefore, an effective and reliable method capable of qualitative and quantitative analysis of the diverse bioactive constituents of ZTPs is required to ensure their safety and efficacy. While significant research has been conducted on the volatile components of Rhizoma Chuanxiong and Radix Angelicae Sinensis, such studies have mainly focused on their cardiovascular, cerebrovascular, neuroprotective, antinociceptive, and anti-inflammatory effects $[22,23]$, and the volatile components of the other ZTPs constituents have not been reported.

In this study, a combined UHPLC coupled with diode array detection (UHPLC-DAD)/GC-MS method was developed to simultaneously identify volatile organic compounds in ZTPs and qualitatively and quantitatively analyze 3 main bioactive constituents present in various ZTPs ingredients. The combined UHPLC-DAD/GC-MS method can be utilized to evaluate the quality of ZTPs.

\section{Experimental}

2.1. Reagents and Chemicals. HPLC grade anhydrous ether and methanol were purchased from Merck (Darmstadt, Germany). Deionized water for the samples and mobile phase were prepared using a Milli-Q50 SP Reagent Water System (Millipore, France). Reference standards for protocatechuic acid, ferulic acid, and ligustilide were obtained from the National Institute for Food and Drug Control (Beijing, China). The purity of all standards was at least 98\%. Thirteen batches of ZTP were purchased from China Resources Sanjiu Medical \& Pharmaceutical Co. Ltd. (Shenzhen, China). Methanol, acetic acid, petroleum ether, ethyl ether, and ethyl acetate were purchased from Baishi Chemical Industry Co. Ltd. (Tianjin, China) and of analytical grade. All solvents were filtered through $0.22 \mu \mathrm{m}$ membrane filters before analysis.

2.2. Sample Preparation. ZTPs were smashed into powder (40 mesh). For HPLC analysis, $3.0 \mathrm{~g}$ of pulverized ZTP was ultrasonically extracted at room temperature with $50 \mathrm{~mL}$ methanol for $0.5 \mathrm{~h}$ in a $250 \mathrm{~mL}$ triangular flask and dried. The ZTP residue was dissolved with $20 \mathrm{~mL}$ water and extracted 3 times with ethyl acetate (30, 30, and $20 \mathrm{~mL})$. The ethyl acetate extracts were dried using an electrothermostatic water bath. Finally, the extract was reconstituted in $5 \mathrm{~mL}$ methanol. For GC-MS analysis, $3.0 \mathrm{~g}$ of pulverized ZTP was reflux-extracted twice for $1 \mathrm{~h}$ at $50^{\circ} \mathrm{C}$ with $20 \mathrm{~mL}$ ethyl acetate in a $250-\mathrm{mL}$ triangular flask. The ethyl acetate extracts were dried and reconstituted in $5 \mathrm{~mL}$ ethyl acetate. All ZTP solutions were filtered through a $0.22 \mu \mathrm{m}$ nylon membrane filter.

2.3. GC-MS Analysis. GC-MS analysis was performed on an Agilent 7890/5975C-GC/MSD instrument (Agilent Technologies, USA) coupled with a HP-5MS fused silica capillary column $(30 \mathrm{~m} \times 0.25 \mathrm{~mm} \times 0.25 \mu \mathrm{m}$; Agilent Technologies, Santa Clara, CA, USA). The GC oven temperature was initially increased from $60^{\circ} \mathrm{C}$ to $120^{\circ} \mathrm{C}$ at a rate of $10^{\circ} \mathrm{C} / \mathrm{min}$, then elevated at a rate of $3^{\circ} \mathrm{C} / \mathrm{min}$ up to $175^{\circ} \mathrm{C}$, then increased at a rate of $5^{\circ} \mathrm{C} / \mathrm{min}$ up to $205^{\circ} \mathrm{C}$, then went up at a rate of $0.8^{\circ} \mathrm{C} / \mathrm{min}$ up to $210^{\circ} \mathrm{C}$, and then raised to $280^{\circ} \mathrm{C}$ at a rate of $5^{\circ} \mathrm{C} / \mathrm{min}$, held for $5 \mathrm{~min}$, giving a total runtime of $55.583 \mathrm{~min} .1 \mu \mathrm{L}$ volume of ethyl acetate extracts was injected into the GC. Helium carrier gas at a constant flow rate of $1.0 \mathrm{~mL} / \mathrm{min}$ and a $30: 1$ split ratio were used simultaneously. Mass spectrometer was operated in full scan with an electron energy of $70 \mathrm{eV}$; interface temperature: $280^{\circ} \mathrm{C}$; MS source temperature: $230^{\circ} \mathrm{C}$; MS quadrupole temperature: $150^{\circ} \mathrm{C}$. The scan range was from $\mathrm{m} / \mathrm{z} 30$ to 550 .

2.4. HPLC Analysis. HPLC analysis was performed on a Thermo Ultimate-3000 system (Thermo Scientific, Waltham, MA, USA) coupled with a DAD. A Phenomenex Kinetex XBC18 column $\left(100 \mathrm{~mm} \times 4.6 \mathrm{~mm} ; 2.6 \mu \mathrm{m}\right.$ i.d.; $25^{\circ} \mathrm{C}$ column temperature $)$ with a guard column $(2.1 \mathrm{~mm} \times 4.6 \mathrm{~mm}, 2.6 \mu \mathrm{m}$ i.d.) was used (Phenomenex, Torrance, CA, USA). The mobile phase was $0.2 \%(\mathrm{v} / \mathrm{v})$ aqueous acetic acid solution (A) and methanol (B). The linear gradient was as follows: $0-7 \mathrm{~min}$, $10 \% \mathrm{~B}$; 7-15 min, $10-15 \% \mathrm{~B} ; 15-35 \mathrm{~min}, 15-40 \% \mathrm{~B}$; $35-65 \mathrm{~min}$, $40-50 \% \mathrm{~B}$. The flow rate was $1.5 \mathrm{~mL} / \mathrm{min}$ and the injection volume was $2 \mu \mathrm{L}$.

2.5. HPLC Method Validation. All components were quantified using chromatograms obtained at $260 \mathrm{~nm}$. The quantification was validated in terms of linearity, limit of detection (LOD), limit of quantification (LOQ), accuracy, and precision.

The stock solution containing the 3 markers was prepared and diluted to appropriate concentration ranges for the establishment of calibration curves. The calibration graphs were plotted after linear regression of the peak areas versus the corresponding concentrations. Good linear behavior was observed, with correlation coefficients $(r)$ between 0.9992 and 0.9994 . LOD and LOQ were determined at signal-to-noise $(S / N)$ ratios of approximately 3 and 10 , respectively. Recovery experiments were performed at medium levels. The concentrations of protocatechuic acid, ferulic acid, and ligustilide were $0.0204 \mathrm{mg} / \mathrm{mL}$, 
TABLE 1: Regression equations, correlation coefficients, linearity ranges, LODs, LOQs, and recoveries of investigated compounds.

\begin{tabular}{lccccccc}
\hline Analytes & Regression equation & $R^{2}$ & Linearity range/ $\mu \mathrm{g} \mathrm{mL} \mathrm{L}^{-1}$ & $\mathrm{LOD} / \mu \mathrm{g}$ & $\mathrm{LOQ} / \mu \mathrm{g}$ & $\mathrm{Recovery} \%$ & $\mathrm{RSD} \%$ \\
\hline Protocatechuic acid & $Y=38.403 X+0.0743$ & 0.9994 & $4.1-28.6$ & 0.0019 & 0.0037 & 100.68 & 0.35 \\
Ferulic acid & $Y=13.889 X+0.3961$ & 0.9992 & $80.2-561.4$ & 0.025 & 0.074 & 100.98 & 0.56 \\
Ligustilide & $Y=9.1687 X+1.1081$ & 0.9994 & $110-770$ & 0.044 & 0.084 & 102.69 & 0.28 \\
\hline
\end{tabular}

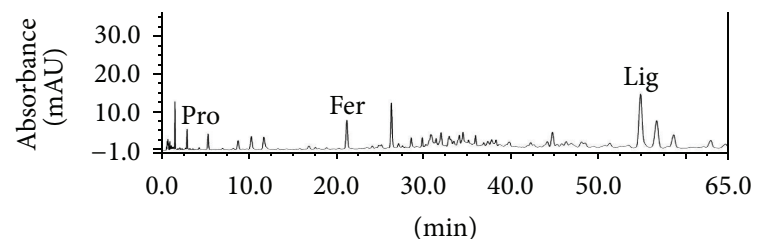

(a)

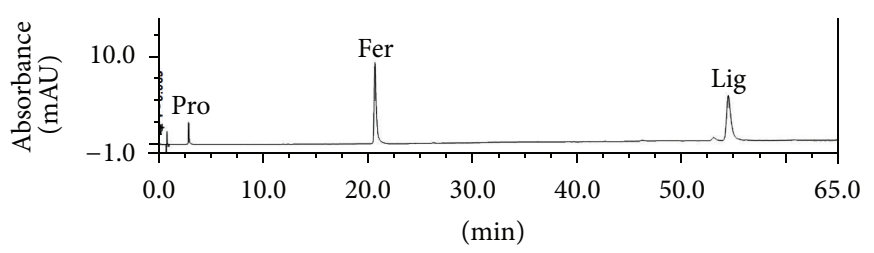

(b)

FIGURE 1: Representative HPLC chromatograms of mixed standards and the ZTP extract at $260 \mathrm{~nm}$. (a) ZTP extract (sample 1209051H); (b) mixed standards of the 3 chemical constituents. Peaks: protocatechuic acid, ferulic acid, and ligustilide.

$0.1027 \mathrm{mg} / \mathrm{mL}$, and $0.22 \mathrm{mg} / \mathrm{mL}$. This procedure was accordingly repeated for six replicates. The spiked samples were extracted, processed, and quantified in accordance with the methods described above. Recoveries varied from 100.18 to $103.39 \%$, with RSDs from 0.50 to $2.36 \%$ (Table 1).

Precision was evaluated with the solution of sample $1209051 \mathrm{H}$ under the selected optimal conditions 6 times in 1 day to measure intraday variation. The RSDs of the precision results were in the range of $0.54-0.89 \%$. Repeatability was confirmed with 6 different working solutions prepared from sample $1209051 \mathrm{H}$. The RSDs of the repeatability results were in the range of $1.88-2.31 \%$. The stability of the solutions was tested by injecting them into the apparatus at $0,2,4,6,8,12$, and $24 \mathrm{~h}$. The RSDs of the stability results were in the range of $1.80-2.53 \%$.

Moreover, specificity was investigated by comparing the chromatograms of mixed standards and the ZTP extract (Figure 1). According to the three-dimensional plot of the absorbance as a function of retention time and wavelength in the HPLC-DAD data for sample number $1209051 \mathrm{H}$, no evidence of peak of impurities overlapping the markers was found.

This is the first simultaneous analysis of protocatechuic acid, ferulic acid, and ligustilide with acceptable linearity, precision, repeatability, and accuracy.

\section{Results and Discussion}

3.1. Optimization of the GC Method and Extraction. In this study, the extraction efficiencies of petroleum ether, ethyl ether, and ethyl acetate were compared. Total ion current (TIC) was greatest when ethyl acetate was used. Investigation of the extraction efficiencies of the reflux and ultrasonic methods indicated that total TIC was higher when the reflux method was used. The GC results for the different solvent extracts and methods are described in Table 2 and Figure 2. In the ethyl acetate solvent extracts, 22 components were identified by artificial analysis and computer retrieval, while relative content was determined by the area normalization method. The main components of the ethyl acetate solvent extracts were ligustilide (19.381\%), oleic acid (10.012\%), 9,12-octadecadienoic acid (9.346\%), butylidene phthalide (5.055\%), dibutyl phthalate $(5.891 \%)$, xanthyletin $(3.813 \%)$, methyl eugenol $(3.833 \%)$, n-docosane (3.545\%), asarinin $(2.085 \%)$, and safrole (1.555\%). In comparison to the ethyl acetate solvent extract samples, the ethyl ether extract samples contained a greater amount of 2,6-di-tert-butyl-p-cresol (2.667\%), while the petroleum ether extract samples contained a greater amount of sulfur (3.993\%).

3.2. Optimization of the HPLC Method and Extraction. Due to the existence of acidic components in the ZTP extraction, a small amount of acid was added to the mobile phase to ease ionization of these components, with the goal of improving peak shape and restraining peak tailing. Different concentrations of acetic acid, phosphoric acid, and formic acid were compared for this purpose. The results showed that all compounds could be baseline-separated when $0.2 \%$ aqueous acetic acid solution was added.

DAD detection was performed within a wavelength range of 190-400 $\mathrm{nm}$. When the chromatograms and characteristic UV spectra of the 3 reference compounds were compared, it was found that the 3 active compounds had higher absorbance, better separation, and a steady baseline at $260 \mathrm{~nm}$ in comparison with the other tested wavelengths.

The extraction procedure was optimized prior to sample analysis. The samples ( $3.0 \mathrm{~g}$ each) were extracted with different volumes and percentages of methanol, as well as different volumes and percentages of ethanol, respectively. The optimum results were obtained using $50 \mathrm{~mL}$ methanol. Investigation of the dependence of the yield on 


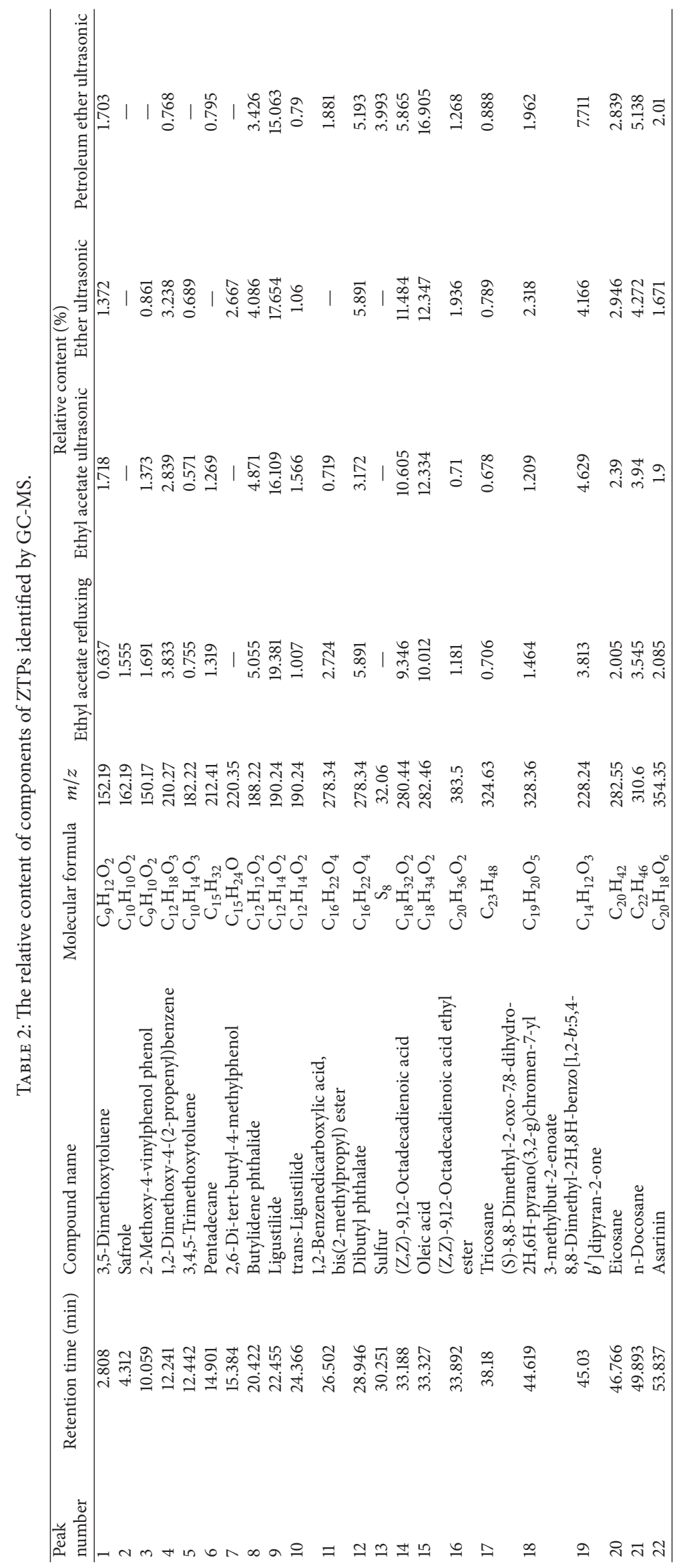




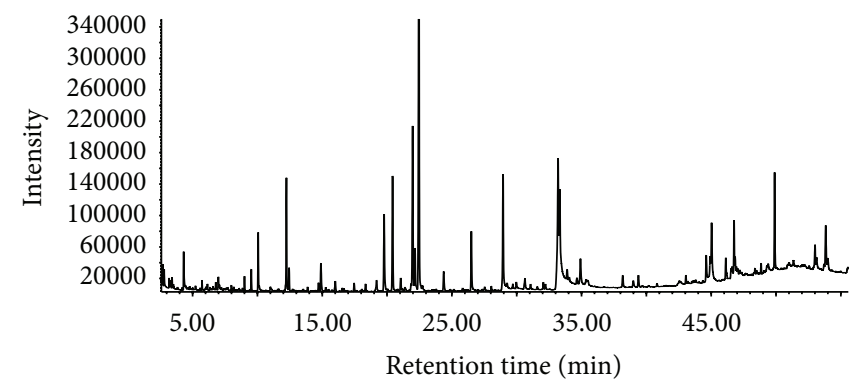

(a)

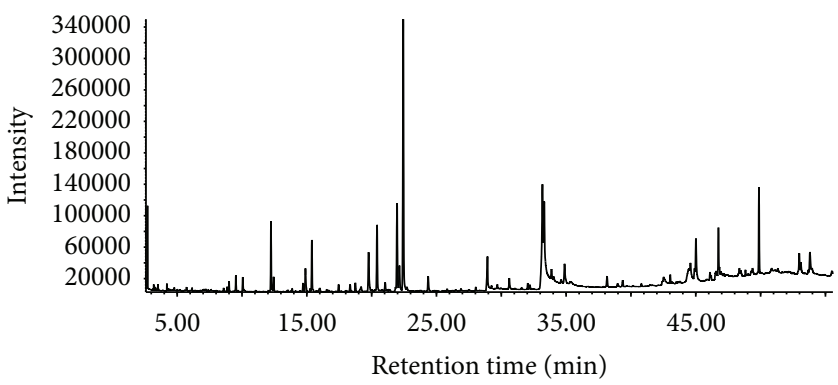

(c)

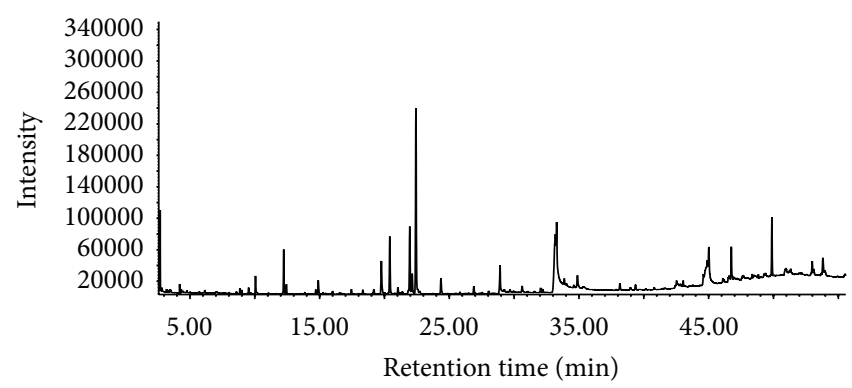

(b)

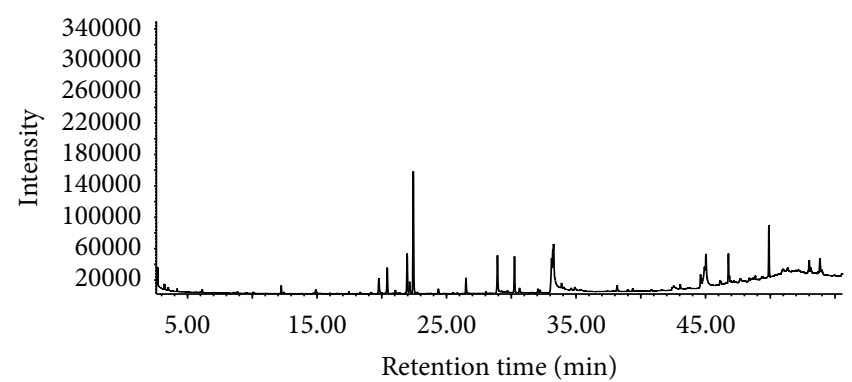

(d)

FIgURE 2: The total ion chromatogram (TIC) of ZTPs using different solvents and extraction methods: (a) ethyl acetate extraction by reflux method, (b) ethyl acetate extraction by ultrasonic wave, (c) ether extraction by ultrasonic wave, and (d) petroleum ether extraction by ultrasonic wave.

the duration of the extraction $(15,30$, and $60 \mathrm{~min})$ showed that all of the investigated compounds were almost completely extracted when 30 min extraction was used.

3.3. Sample Analysis. The UHPLC-DAD/GC-MS method was applied to analyze 13 batches of ZTP (Tables 3 and 4). Protocatechuic acid content ranged from 6.07 to $17.30 \mu \mathrm{g} / \mathrm{g}$, ferulic acid content ranged from 79.59 to $115.90 \mu \mathrm{g} / \mathrm{g}$, and ligustilide content ranged from 79.59 to $388.69 \mu \mathrm{g} / \mathrm{g}$. The GC-MS results showed that the average relative contents of ligustilide, oleic acid, asarinin, safrole, 2-methoxy-4-vinylphenol, and 1,2-dimethoxy-4-(2propenyl)benzene were $6.991 \%, 23.275 \%, 1.631 \%, 0.586 \%$, $0.575 \%$, and $0.574 \%$, respectively. 3,5-Dimethoxytoluene was not detected. These results suggested that the contents of each component varied greatly among batches of ZTPs. The largest observed difference in content was nearly 5 -fold, indicating large variations in the ZTP production process and/or incorrectly identified herbal sources.

The UHPLC-DAD/GC-MS method we established could detect as much volatile components as possible. Compared to previous study using a two-dimensional liquid chromatography coupled to mass spectrometry, there were more compounds identified by the GC-MS method, such as 1,2-dimethoxy-4-(2-propenyl)benzene, 2-methoxy4 -vinylphenol phenol, butylidene phthalide, oleic acid, and (Z,Z)-9,12-octadecadienoic acid ethyl ester, which were mainly from Rhizoma Chuanxiong [24]. It is very helpful for a comprehensive understanding of volatile components of ZTPs. At the same time, the established UHPLC-DAD method could determine protocatechuic acid, ferulic acid, and ligustilide simultaneously, which was very helpful and practicable for the quality control of ZTPs for companies. Since TCM is a complex system containing tens or even hundreds of different chemical constituents, the active compounds of most TCM still remain unknown. It is reported that molecular biochromatography was a novel strategy for the screening and analysis of biologically active compounds in TCM $[25,26]$. Thus, in our further study, we will make an attempt to screen active compounds of ZTPs by using molecular biochromatography.

\section{Conclusions}

A UHPLC-DAD/GC-MS method was established for the comprehensive analysis of ZTPs that allowed separation of complex constituents in a short time. GC-MS provided accurate masses of protonated molecules, which were helpful for compound identification. The UHPLC-DAD/GC-MS method was successfully applied for simultaneous determination of 3 bioactive compounds in ZTP. The UHPLC$\mathrm{DAD} / \mathrm{GC}-\mathrm{MS}$ method is readily available, rapid, and reliable. Therefore, the UHPLC-DAD/GC-MS method is suitable for routine analysis, original discrimination, and effective quality control of ZTPs. The amounts of protocatechuic acid, ferulic acid, and ligustilide in the ZTP batches varied considerably. 


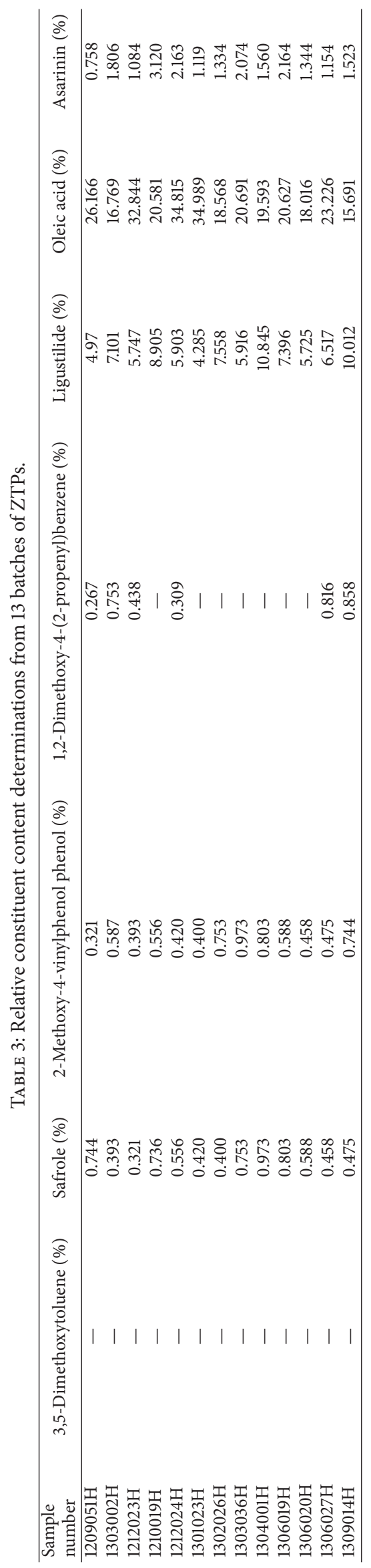


TABLE 4: Determination of the contents of protocatechuic acid, ferulic acid, and ligustilide of 13 batches of ZTPs.

\begin{tabular}{|c|c|c|c|c|c|c|}
\hline \multirow{3}{*}{ Sample number } & \multicolumn{6}{|c|}{ Content $(\mu \mathrm{g} / \mathrm{g})$} \\
\hline & \multicolumn{2}{|c|}{ Protocatechuic acid } & \multicolumn{2}{|c|}{ Ferulic acid } & \multicolumn{2}{|c|}{ Ligustilide } \\
\hline & Mean & RSD (\%) & Mean & RSD (\%) & Mean & RSD (\%) \\
\hline $1209051 \mathrm{H}$ & 14.01 & 1.91 & 80.11 & 2.78 & 174.13 & 1.65 \\
\hline $1303002 \mathrm{H}$ & 14.43 & 1.26 & 79.59 & 1.42 & 79.59 & 2.52 \\
\hline $1303002 \mathrm{H}$ & 17.30 & 1.39 & 80.37 & 1.84 & 178.85 & 2.65 \\
\hline $1210019 \mathrm{H}$ & 8.36 & 0.82 & 78.06 & 2.02 & 363.42 & 0.39 \\
\hline $1212024 \mathrm{H}$ & 10.64 & 2.75 & 82.51 & 1.21 & 348.78 & 2.48 \\
\hline $1301023 \mathrm{H}$ & 12.21 & 1.25 & 86.42 & 0.88 & 252.37 & 2.57 \\
\hline $1302026 \mathrm{H}$ & 10.08 & 1.67 & 115.90 & 2.62 & 323.11 & 2.47 \\
\hline $1303036 \mathrm{H}$ & 6.07 & 2.51 & 84.98 & 3.00 & 388.69 & 1.05 \\
\hline $1304001 \mathrm{H}$ & 10.02 & 1.61 & 111.97 & 2.35 & 268.61 & 2.42 \\
\hline $1306019 \mathrm{H}$ & 9.62 & 1.61 & 86.68 & 1.32 & 303.62 & 0.81 \\
\hline $1306020 \mathrm{H}$ & 8.13 & 1.26 & 80.93 & 2.89 & 355.71 & 1.72 \\
\hline $1306027 \mathrm{H}$ & 9.71 & 0.37 & 93.14 & 0.49 & 260.75 & 0.52 \\
\hline $1309014 \mathrm{H}$ & 9.64 & 2.86 & 96.50 & 2.89 & 301.90 & 0.59 \\
\hline
\end{tabular}

Therefore, future studies should focus on evaluating the influence of bioactive constituent of ZTPs on their therapeutic effects using preclinical pharmacodynamic and clinical testing.

\section{Conflict of Interests}

The authors declare that there is no conflict of interests.

\section{Acknowledgments}

This work was supported by the Chinese Medicinal Materials Production and Construction Projects of the Ministry of Industry and Information Technology of China ([2015] no. 282) and the Collaborative Innovation Center Research Team Construction Project in Guangdong Province-the Innovation Research Team of Traditional Chinese Medicine Resources (A1-AFD01514A04).

\section{References}

[1] Kinetex core-shell particles column, G. F. S. I. C., http://www .gzflm.com/productinfo/detail_4_6_74.aspx.

[2] R. Hayes, A. Ahmed, T. Edge, and H. Zhang, "Core-shell particles: preparation, fundamentals and applications in high performance liquid chromatography," Journal of Chromatography A, vol. 1357, pp. 36-52, 2014.

[3] A. Natale, D. Nardiello, C. Palermo, M. Muscarella, M. Quinto, and D. Centonze, "Development of an analytical method for the determination of polyphenolic compounds in vegetable origin samples by liquid chromatography and pulsed amperometric detection at a glassy carbon electrode," Journal of Chromatography A, vol. 1420, pp. 66-73, 2015.

[4] R. Preti, M. L. Antonelli, R. Bernacchia, and G. Vinci, "Fast determination of biogenic amines in beverages by a core-shell particle column," Food Chemistry, vol. 187, pp. 555-562, 2015.
[5] E. A. Nonato, F. Carazza, F. C. Silva, C. R. Carvalho, and Z. D. L. Cardeal, "A headspace solid-phase microextraction method for the determination of some secondary compounds of Brazilian sugar cane spirits by gas chromatography," Journal of Agricultural and Food Chemistry, vol. 49, no. 8, pp. 3533-3539, 2001.

[6] T. Górecki, J. Harynuk, and O. Panić, "The evolution of comprehensive two-dimensional gas chromatography (GC $\times \mathrm{GC})$," Journal of Separation Science, vol. 27, no. 5-6, pp. 359-379, 2004.

[7] F. Gong, Y.-Z. Liang, H. Cui, F.-T. Chau, and B. T.-P. Chan, "Determination of volatile components in peptic powder by gas chromatography-mass spectrometry and chemometric resolution," Journal of Chromatography A, vol. 909, no. 2, pp. 237-247, 2001.

[8] S. Shen, Y. Sha, C. Deng, X. Zhang, D. Fu, and J. Chen, "Quality assessment of Flos Chrysanthemi Indici from different growing areas in China by solid-phase microextraction-gas chromatography-mass spectrometry," Journal of Chromatography A, vol. 1047, no. 2, pp. 281-287, 2004.

[9] C. Deng, Y. Mao, N. Yao, and X. Zhang, "Development of microwave-assisted extraction followed by headspace solid-phase microextraction and gas chromatography-mass spectrometry for quantification of camphor and borneol in Flos Chrysanthemi Indici," Analytica Chimica Acta, vol. 575, no. 1, pp. 120-125, 2006.

[10] C. Deng, N. Yao, B. Wang, and X. Zhang, "Development of microwave-assisted extraction followed by headspace singledrop microextraction for fast determination of paeonol in traditional Chinese medicines," Journal of Chromatography A, vol. 1103, no. 1, pp. 15-21, 2006.

[11] H. Zhu, Y. Wang, H. Liang, Q. Chen, P. Zhao, and J. Tao, "Identification of Portulaca oleracea L. from different sources using GC-MS and FT-IR spectroscopy," Talanta, vol. 81, no. 1-2, pp. 129-135, 2010.

[12] L. Tong, Y. Wang, J. Xiong, Y. Cui, YigangZhou, and L. Yi, "Selection and fingerprints of the control substances for plant drug Eucommia ulmodies Oliver by HPLC and LC-MS," Talanta, vol. 76, no. 1, pp. 80-84, 2008. 
[13] G.-H. Ruan and G.-K. Li, “The study on the chromatographic fingerprint of Fructus xanthii by microwave assisted extraction coupled with GC-MS," Journal of Chromatography B: Analytical Technologies in the Biomedical and Life Sciences, vol. 850, no. 1-2, pp. 241-248, 2007.

[14] State Pharmacopoeia Commission of the People's Republic of China, Pharmacopoeia of People's Republic of China, Chemical Industry Press, Beijing, China, 2010.

[15] H. R. Qian, X. K. Qi, G. Huang, and D. G. Feng, "Comparison of effect of zhengtian pill and tongtian oral solution in treating tension-type headache," Chinese Journal of New Drugs and Clinical Remedies, vol. 22, no. 5, pp. 279-282, 2003.

[16] Z. Xie, W. L. Li, and G. J. Shi, "Randomized, double-blind, dontrolled clinical study of Zhengtianwan pill in treating 42 cases of migraine," Journal of Mathematical Medicine, vol. 24, no. 1, pp. 70-73, 2011.

[17] C. X. Y. Y. Wang, H. C. Shang, M. Ren, and J. Yuan, “doubleblind and double-dummy, multi-center clinical study of Zhengtian Pill for patients with migraine," Chinese Traditional Patent Medicine, vol. 34, no. 5, pp. 791-794, 2012.

[18] L. Huang, H.-L. Chen, and L.-L. Li, "Simultaneous determination of paeoniflorin, ferulic acid, prim-O-glucosylcimifugin and $4^{\prime}$-O-beta-glucopyranosyl-5-O-methylvisamminol in Zhengtian pills by HPLC," Zhongguo Zhongyao Zazhi, vol. 38, no. 13, pp. 2114-2117, 2013.

[19] A. G. Z. Q. Lin, J. N. Chen, X. P. Lai, S. H. Gui, and C. P. Fang, "Anti-inflammatory and analgesic effects of ligustilide," Chinese Journal of Experimental Traditional Medical Formulae, vol. 17, no. 77, pp. 165-168, 2011.

[20] J. M. L. M. Zhai, R. An, F. L. Wu, and H. H. Xu, "Content determination of protocatechuic acid in Spatholobus suberectus dumn," Traditional Chinese Drug Research \& Clinical Pharmacology, vol. 20, no. 5, pp. 462-465, 2009.

[21] X. Y. X. Y. Y. Hu, "Chemical and pharmacological research progress of ferulic acid," Chinese Traditional Patent Medicine, vol. 28, no. 2, pp. 253-255, 2006.

[22] X.-P. Chen, W. Li, X.-F. Xiao, L.-L. Zhang, and C.-X. Liu, "Phytochemical and pharmacological studies on Radix Angelica sinensis," Chinese Journal of Natural Medicines, vol. 11, no. 6, pp. 577-587, 2013.

[23] X. Ran, L. Ma, C. Peng, H. Zhang, and L.-P. Qin, "Ligusticum chuanxiong Hort: a review of chemistry and pharmacology," Pharmaceutical Biology, vol. 49, no. 11, pp. 1180-1189, 2011.

[24] X. Chen, L. Kong, X. Su et al., "Separation and identification of compounds in Rhizoma chuanxiong by comprehensive twodimensional liquid chromatography coupled to mass spectrometry," Journal of Chromatography A, vol. 1040, no. 2, pp. 169-178, 2004.

[25] H. Wang, L. Kong, H. Zou, J. Ni, and Y. Zhang, "Screening and analysis of biologically active compounds in Angelica sinensis by molecular biochromatography," Chromatographia, vol. 50, no. 7-8, pp. 439-445, 1999.

[26] H. Wang, H. Zou, L. Kong, J. Ni, and Y. Zhang, "Screening and analysis of biologically active components in traditional Chinese medicine by molecular biochromatography," Chinese Journal of Chromatography, vol. 17, no. 2, pp. 123-127, 1999. 

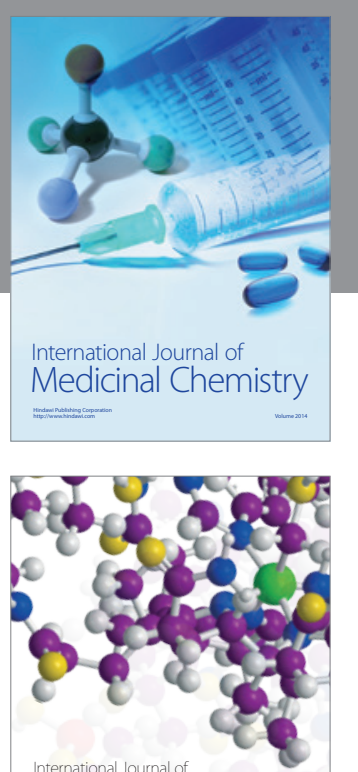

Carbohydrate Chemistry

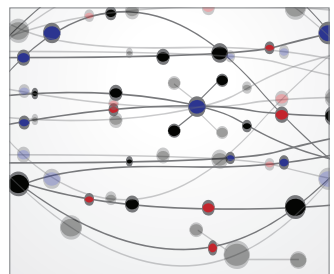

The Scientific World Journal
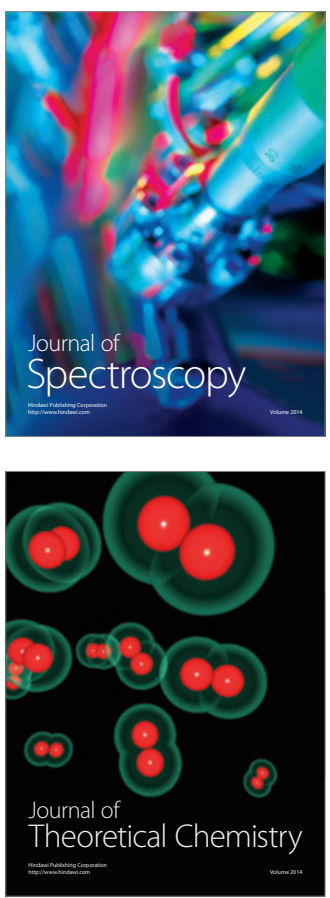
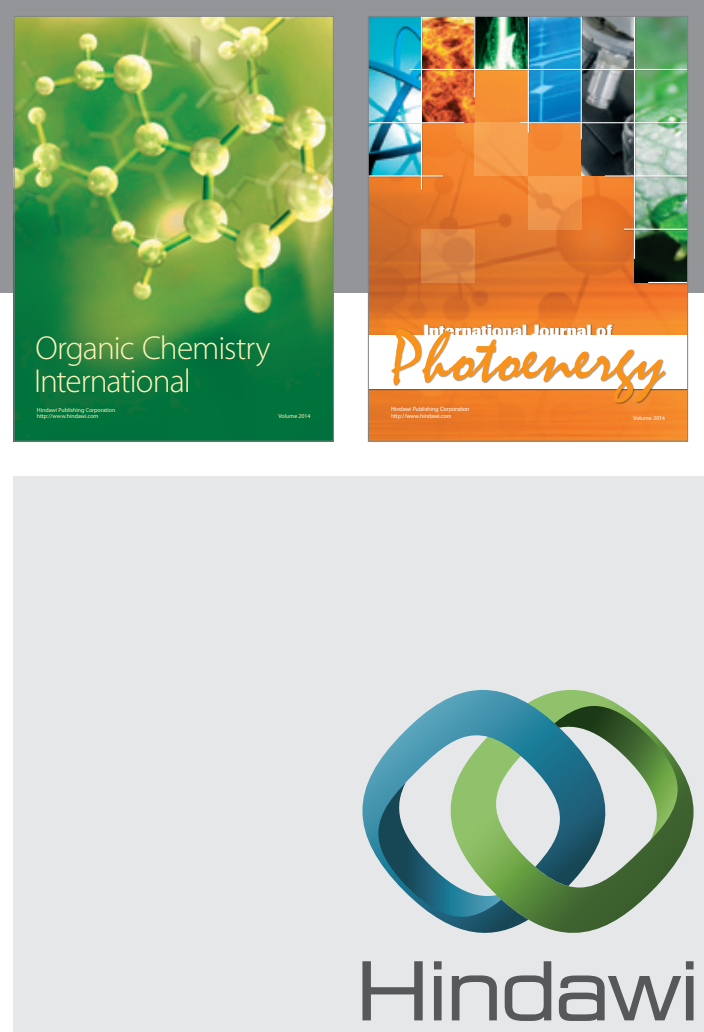

Submit your manuscripts at

http://www.hindawi.com

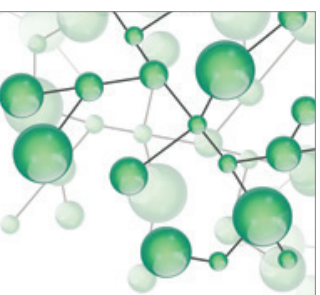

International Journal of

Inorganic Chemistry

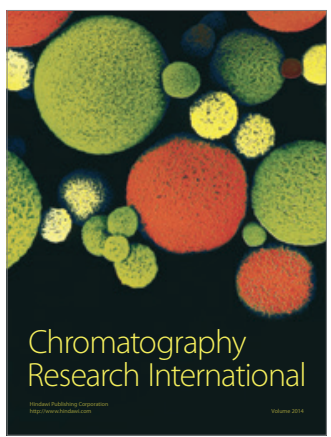

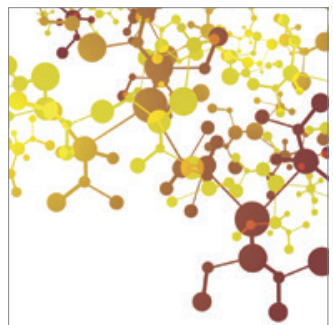

Applied Chemistry
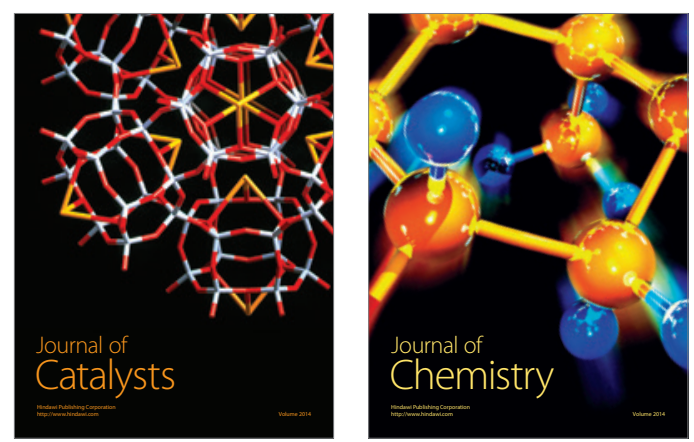
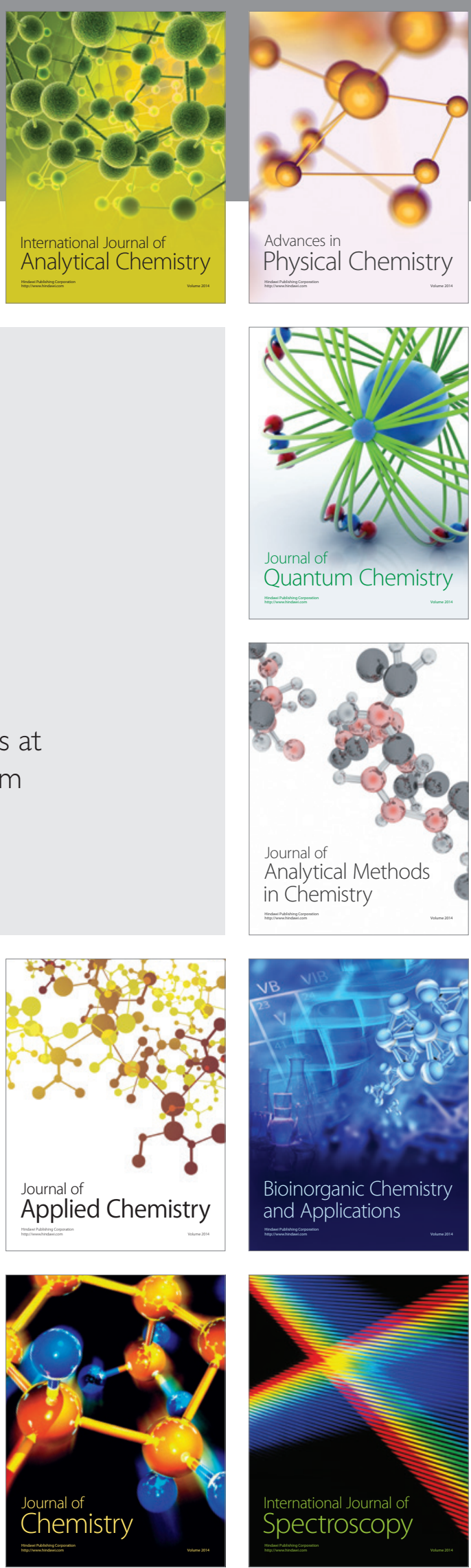